\title{
Surgeon Preference May Be More Important Than Models of Care When It Comes to Early Laparoscopic Cholecystectomy Rates for Acute Cholecystitis
}

\author{
Daniel Feng ${ }^{1,2, ~ *, ~ L u k e ~ P e t s c h a c k ~}{ }^{1,2}$, Georgia Marr², Jon Gani ${ }^{1,2}$ \\ ${ }^{1}$ School of Medicine and Public Health, University of Newcastle, Newcastle, Australia \\ ${ }^{2}$ Department of Surgery, John Hunter Hospital, Newcastle, Australia
}

Email address:

d.feng1996@gmail.com (D. Feng)

${ }^{*}$ Corresponding author

To cite this article:

Daniel Feng, Luke Petschack, Georgia Marr, Jon Gani. Surgeon Preference May Be More Important Than Models of Care When It Comes to Early Laparoscopic Cholecystectomy Rates for Acute Cholecystitis. Journal of Surgery. Vol. 8, No. 6, 2020, pp. $228-232$.

doi: $10.11648 /$ j.js. 20200806.20

Received: November 16, 2020; Accepted: December 7, 2020; Published: December 22, 2020

\begin{abstract}
Aim: Early Laparoscopic Cholecystectomy (ELC) for acute cholecystitis is widely accepted as the standard of care. The capacity to deliver this has been strongly linked to the establishment of Acute Surgical Units (ASU). This study aimed to determine the relative effects of surgeon preference on ELC rates. Method: A retrospective audit of patients with acute cholecystitis was carried out over 6 months in 3 hospitals in 2018. One hospital had an ASU and 2 hospitals had no ASU. The timing of cholecystectomy, intraoperative cholangiogram rates and length of hospital stay were collected. Results: 175 patients were included; 92 admitted to the ASU hospital and 83 admitted to non-ASU hospitals. When adjusted for severity, the ELC rate was $62 \%$ and $31 \%(\mathrm{P}<0.0001)$ in the ASU hospital and non-ASU hospitals respectively in patients with mild (Tokyo Grade I) disease. There was no difference between intraoperative cholangiogram rates between hospitals. The initial length of stay was on average 2.4 days shorter in the early ELC patients ( $\mathrm{MD}=-2.4,95 \% \mathrm{CI} 1.3$ to 3.4). The 2 Non-ASU hospitals varied significantly in ELC rates $(19 \%$ and $48 \% \mathrm{P}=0.0158)$, the hospital with the higher ELC rates shared senior surgical staff with the ASU hospital. Conclusion: Hospitals with an ASU are better able to provide timely surgery to patients presenting with acute cholecystitis and this is associated with a reduction of time in hospital for these patients, but surgeon preference may be more important in determining ELC rates than the ASU model of care.
\end{abstract}

Keywords: Early Laparoscopic Cholecystectomy, Surgeon Preference, Acute Cholecystitis

\section{Introduction}

Laparoscopic cholecystectomy has become the mainstay of treatment for acute cholecystitis. However, the timing of surgery can be divided into 'early' and 'delayed'.

The definition of early laparoscopic cholecystectomy (ELC) differs in the literature from anywhere between less than 24 hours after admission to less than 7 days after admission. However the most common definition is commencement of laparoscopic cholecystectomy within 72 hours of the patient's admission to hospital [1-3], and this is the definition of ELC used in this study.

Although there are no current Australian-specific guidelines for the management of acute cholecystitis, the international stance favours ELC for patients presenting with acute cholecystitis in most cases. The 2014 National Institute for Health and Care Excellence guidelines recommend surgery as early as possible after admission and the Tokyo Guidelines 2018 (TG18) recommend surgery be carried out as early as possible regardless of time of onset, ideally within 72 hours of presentation $[2,4]$.

The other option for the surgical management of acute cholecystitis is delayed laparoscopic cholecystectomy (DLC), treating acute cholecystitis conservatively, and performing an interval cholecystectomy at a later date when the acute inflammation has settled. Historically surgeons preferred DLC over ELC for its perceived increased safety. The concern regarding ELC stems from the theoretically increased risk of bile 
duct injury and conversion to open surgery due to distorted anatomy in the acutely inflamed state $[5,6]$.

However, the current literature suggests that ELC is at least as safe as DLC when comparing post-operative complications and conversion to open surgery [2, 3, 5-9], and results in less re-presentations for gallbladder related pathology, and an overall shorter length of hospital stay and lower cost to the health system $[2,3,6-8,10]$.

Despite this evidence, surgical practice internationally does not reflect this recommendation. De Mestral et al notes that the rate of ELC is between 36-88\% in UK, Japan and $U^{3}{ }^{3}$. Kao et al reports similar statistics where emergency cholecystectomy was performed in only $53 \%$ of acute cholecystitis cases in the US, and $16 \%$ in the $\mathrm{UK}^{11}$. The lack of uptake of ELC given the evidence suggests multiple barriers for adoption. Logistical difficulties were expressed in multiple studies $[1,4,11,12]$. Redesigning services, such as the advent of Acute Surgical Unit (ASU) models in combination with surgeon and organisational level changes have been suggested for the implementation of ELC as a treatment strategy $[4,11]$. The literature shows that the implementation of an ASU model can markedly improve ELC rates [13,14] and these changes can be sustained [15].

While the literature is focused on systemic changes and logistical changes, there is a dearth of evidence exploring the impact of surgeon preference on ELC rates, independent of ASU models of care. ELC rates in 3 metropolitan hospitals in one of the largest Local Health Districts in New South Wales, Australia were compared. Two of the study hospitals are staffed by the same General Surgeons, one hospital has a wellestablished ASU and another has no ASU. In contrast, the third hospital has no formal ASU, and has a completely different group of General Surgeons on staff. We sort to explore how both the effect of an ASU and surgeon preference can affect early laparoscopic cholecystectomy rates.

\section{Method}

\subsection{Study Design}

A retrospective audit was carried out for all cases of Acute Cholecystitis (AC) over the 6-month period July $1^{\text {st }}$ and December $31^{\text {st }} 2018$ using patient medical records from the 3 participating hospitals.

\subsection{Setting}

The 3 hospitals are part of the Hunter New England Local Health District in NSW, Australia and serve a largely metropolitan population. The local health district in question is one of the largest in NSW with a catchment area of over 130,000 square kilometres and an estimated population exceeding 920,000. The 3 study hospitals have been deidentified and labelled as Hospitals A, B and C. Hospital A has a well-established ASU, and Hospitals B and C have no ASU. Of note, Hospital A and Hospital C share the same general surgeons, who operate regularly at both locations.

Hospital A has a well-established ASU with dedicated
ASU consultants (with no other commitments and onsite from $8 \mathrm{am}$ until $7 \mathrm{pm}$ ) and dedicated onsite registrars, with emergency operating sessions every afternoon, solely for emergency cases, including acute cholecystitis. Hospital B does not have an ASU as such, however there is a hybrid system in place, the on-call surgeon will fill $70 \%$ of their operating list with elective cases during daytime hours, with a small portion of the list (usually about 3 hours) left for any emergency cases. Hospital $\mathrm{C}$ has no ASU, and emergency cases are usually done on an ad hoc basis after elective cases, or rarely these cases may interrupt elective lists if they are very urgent.

\subsection{Participants}

Study subjects are all adult patients presenting to the study hospitals with acute cholecystitis, diagnosed as per the Tokyo Guidelines 2018 criteria [16]. Exclusion criteria were; paediatric patients, patients who discharged against medical advice, and patients transferred to a private hospital for management, as data could not be accessed.

\subsection{Data Collection}

Subjects were identified by searching the clinical coding database of the study hospitals. The records were searched electronically for all the potentially relevant ICD 10 Codes (see Appendix 1). Patient files were manually reviewed to include those patients with a clinical diagnosis of acute cholecystitis as per the TG18 Guidelines [16]. The relevant data for outcome measures was collected from the medical records.

\subsection{Outcome Measures}

The primary outcome measure was time from admission to cholecystectomy. Secondary outcomes included; intraoperative cholangiogram rates and length of hospital stay.

\subsection{Statistical Analysis}

The data was analysed using R (version 3.6.3). Students' unpaired T-test was used for comparison of categorical and continuous data. A P-value of $<0.05$ was considered statistically significant. Ethics Approval.

The project was authorised to proceed as a non-research activity by the HNELHD-HREC committee on the $1^{\text {st }}$ of March, 2019. Authorisation Number: AU201903-01.

\section{Results}

175 unique presentations of acute cholecystitis were recorded at the study hospitals in the six-month study period. Of those, ninety-two patients presented to Hospital A (ASU), fifty-two patients presented to Hospital B (Non-ASU), and thirty-one presented to Hospital C (Non-ASU), for a total of ninety-two in the ASU group and eighty-three in the nonASU group. The severity of AC by Tokyo Grade is shown in Table 1 and is comparable. 


\subsection{Primary Outcome}

The early laparoscopic cholecystectomy (ELC) rate at these hospitals differed significantly; $59 \%$ of patients admitted with Acute Cholecystitis underwent a Cholecystectomy within 72 hours in the ASU hospital compared with $34 \%$ in the non-ASU hospitals $(\mathrm{P}=0.0008)$

A subgroup analysis was performed for patients who had Tokyo Grade I acute cholecystitis. The early laparoscopic cholecystectomy rate was $62 \%$ and $31 \%(\mathrm{P}<0.0001)$ in the ASU group and non-ASU group respectively.

Subgroup analysis comparing Hospital A with an ASU (77 patients) and Hospital B with no ASU (37 patients) for patients with Tokyo Grade 1 Acute Cholecystitis showed a highly significant difference in ELC rates $(62 \%$ vs $19 \%, \mathrm{P}<0.0001)$.

However, subgroup analysis for patients with Tokyo Grade 1 Acute Cholecystitis between Hospital A (ASU, 77 patients) and Hospital C (No ASU but same surgeons, 25 patients) showed the early laparoscopic cholecystectomy rate was not significantly different ( $62 \%$ and $48 \%$ respectively, $\mathrm{P}=0.1512$ ).

When comparison between the 2 hospitals without ASUs was made for patients with Tokyo Grade 1 Acute Cholecystitis, Hospital B (37) and Hospital C (25), this showed the early laparoscopic cholecystectomy rate differed significantly at $19 \%$ and $48 \%$ respectively $(\mathrm{P}=0.0158)$.

It is worth noting, although the numbers are small, that in Tokyo Grade 2 patients Hospital A and B had similar ELC rates $(45 \%$ and $57 \%$ respectively $\mathrm{P}=0.6951)$ suggesting that in the sicker patients, surgeons at Hospital B are more inclined to operate early.

\subsection{Secondary Outcomes}

There was no significant difference between intraoperative cholangiogram rates between Hospital A, B, and C (94\% vs $87 \%$ vs $94 \%, \mathrm{P}=0.5291)$. The length of stay was on average 2.4 days shorter in the ELC group $(\mathrm{MD}=-2.4,95 \% \mathrm{CI} 1.3$ to 3.4).

\section{Discussion}

The benefits of early laparoscopic cholecystectomy seem clear. ELC compares favourably to interval cholecystectomy in terms of cost to the health system, patient outcomes, and is not inferior in terms of safety $[2,3$, 5-9]. However, Okamoto et al [2] and Gurusamy et $\mathrm{al}^{6}$ are two meta-analyses that highlight an important concern of the high risk of bias. The examined trials had relatively low sample sizes, poor blinding and because surgical complications occur rarely, confidence intervals were wide. The 2014 NICE guidelines also note that the literature was of low-moderate quality [4].

ELC rates can be considered as measures of the models of care provided for emergency surgical cases but they also reflect surgeon's preference. This study compared one ASU Hospital with two non-ASU hospitals. All three hospitals are situated in and around Newcastle NSW, Australia, and are classed as metropolitan hospitals. Both non-ASU hospitals are within a thirty-five-minute distance to the ASU centre via road transport.

This data has shown that a hospital with an ASU is better able to deliver ELC compared to a hospital without an ASU. However, the severity of acute cholecystitis must be taken into account. Patients with severe disease and displaying signs of multi-organ failure may require significant preoperative preparation or initial treatment by interventional radiology and as such comprise a group of patients who may not be suitable for ELC. The TG18 guidelines published a score for risk-stratifying patients with acute cholecystitis known as the Tokyo Grade [2]. We therefore carried out a subgroup analysis of patients with Tokyo Grade I disease as the recommendation for ELC is strongest in this group and this provided comparable disease across the 3 hospitals. The results show that the ASU Hospital significantly outperformed the non-ASU hospitals as a group.

Two additional subgroup analyses were carried out between Hospital $\mathrm{C}$ and Hospital $\mathrm{A}$ as well as Hospital $\mathrm{C}$ and Hospital B. Hospital C and A were compared as Hospital C does not have an ASU while Hospital A does. However, the two hospitals share consultant surgeons; all the general surgeons at Hospital $\mathrm{C}$ work at Hospital A where they do ASU on call work. Therefore, it offers a unique opportunity to determine whether surgeon preference influences ELC rates independent of the existence of an ASU. There was no significant difference in ELC rates demonstrated between these two hospitals. The comparison of 2 Non-ASU hospitals (Hospital C and Hospital B- which shares no General Surgeons with Hospital A) seems to support this. The fact that there was a significant difference in ELC rate in favour of Hospital C, strongly suggests that surgeon preference is at least as important in determining ELC rates as the presence of an established ASU system of care.

Current literature focuses on the benefits of an ASU based model of care as a way to improve time-to-surgery for acute general surgery cases. Rightly so, because the ASU model improves operating theatre and junior surgical staff availability and allows improved consultant oversight for the diagnosis and management of emergency surgery cases [17]. Arguably the ASU high caseload environment promotes the development of the skills and confidence in ELC that have been shown to be associated with better patient outcomes [18, 19].

However, individual surgeon preference should be viewed as a factor that needs to be considered. Hospital $\mathrm{C}$ without an ASU was able to deliver a similar rate of ELC in Tokyo Grade 1 patients to the ASU hospital. In contrast the surgeons at Hospital B without an ASU were only able to perform ELC in $19 \%$ of Tokyo grade 1 patients cholecystitis. This implies that individual surgeon preference may be more important as the model of care, as Hospital $\mathrm{C}$ is staffed by surgeons who would be accustomed to performing ELC from their experience at the ASU hospital. However, the fact that ELC rates are not significantly different at Hospitals A and B in the Tokyo Grade 2 patients suggests that factors other than surgical expertise are at play.

Intraoperative cholangiogram rates were also explored as 
they are strongly recommended in the TG18 [2], as a way of minimising bile duct injury in laparoscopic cholecystectomies. All hospitals performed well with no statistical differences in cholangiography rates (Hospital A and C 94\%, Hospital B 87\%).

Length of hospital stay was also compared, collecting data for the initial presentation only, and not including additional length of stay for patients returning for interval cholecystectomy or related complications. It showed that ELC patients stayed significantly less days than their nonELC counterparts, 3.44 compared to 5.55 days, which agrees with other available international evidence [2, 3, 6-8].

The limitations of this study are the short time period of the audit, the retrospective nature of the data and the fact that patients were not completely matched for comorbidities (although a comparison of grade 1 only patients is matching for severity of disease). If patients were matched using P-Possum, NSQIP or other operative risk scores, it would have eliminated the patient's pre-morbid state as a confounding factor in the clinical decision-making process for performing ELC.

\section{Conclusion}

Acute Surgical Units provide the environment to facilitate Early Laparoscopic Cholecystectomy (ELC) for Acute Cholecystitis. However, it is clear that surgeon preference makes a significant difference to the rate of ELC even when ASU facilities are not available. It is clear that in order to better adhere to evidence-based practice in the management of acute cholecystitis, other elements of surgeon behaviour need to be considered in addition to systems and models of care.

\section{Appendix}

1. K8000 - Calculus of gallbladder with acute cholecystitis, without mention of obstruction

2. K8001 - Calculus of gallbladder with acute cholecystitis, with obstruction

3. K8010 - Calculus of gallbladder with other cholecystitis, without mention of obstruction

4. K8011 - Calculus of gallbladder with other cholecystitis, with obstruction

5. K8030 - Calculus of bile duct with cholangitis, without mention of obstruction

6. K8031 - Calculus of bile duct with cholangitis, with obstruction

7. K8040 - Calculus of bile duct with cholecystitis, without mention of obstruction

8. K8041 - Calculus of bile duct with cholecystitis, with obstruction

9. K810 - Acute cholecystitis

10. K811 - Pancreatitis, Chronic cholecystitis

11. K819 - Acute Cholecystitis

12. K830 - Cholangitis

13. K850 - Idiopathic acute pancreatitis

14. K851 - Biliary acute pancreatitis

15. K859 - Acute pancreatitis, unspecified

16. U832 - Pancreatitis

Table 1. Patient distribution by severity.

\begin{tabular}{llll}
\hline & Tokyo Grade I & Tokyo Grade II & Tokyo Grade III \\
\hline ASU & $84 \%$ & $12 \%$ & $4 \%$ \\
Non-ASU & $75 \%$ & $23 \%$ & $2 \%$ \\
\hline
\end{tabular}

Table 2. Individual Hospital ELC proportion.

\begin{tabular}{|c|c|c|c|c|c|c|c|c|c|}
\hline & \multicolumn{3}{|c|}{ Tokyo Grade I } & \multicolumn{3}{|c|}{ Tokyo Grade II } & \multicolumn{3}{|c|}{ Tokyo Grade III } \\
\hline & Hospital A & Hospital B & Hospital C & Hospital A & Hospital B & Hospital C & Hospital A & Hospital B & Hospital C \\
\hline ELC (<72hrs) & 48 & 7 & 12 & 5 & 8 & 1 & 1 & 0 & 0 \\
\hline Non-ELC $(>72 \mathrm{hrs})$ & 29 & 30 & 13 & 6 & 6 & 4 & 3 & 1 & 1 \\
\hline Totals & 77 & 37 & 25 & 11 & 14 & 5 & 4 & 1 & 1 \\
\hline ELc Rate & $62 \%$ & $19 \%$ & $48 \%$ & $45 \%$ & $57 \%$ & $20 \%$ & $25 \%$ & $0 \%$ & $0 \%$ \\
\hline
\end{tabular}

Table 3. Overall ELC rate and IOC rate.

\begin{tabular}{llll}
\hline & Overall ELC Rate & Tokyo Grade I ELC Rate & Intraoperative Cholangiogram Rate \\
\hline Hospital A & $59 \%$ & $62 \%$ & $94 \%$ \\
Hospital B & $29 \%$ & $19 \%$ & $87 \%$ \\
Hospital C & $42 \%$ & $48 \%$ & $94 \%$ \\
\hline
\end{tabular}


Table 4. Length of Stay.

\begin{tabular}{ll}
\hline & Mean Length of Stay (days) \\
\hline ELC & 3.44 \\
Non-ELC & 5.80 \\
\hline
\end{tabular}

\section{Acknowledgements}

We would like to acknowledge Dr Jessie Gibson, Dr Josh O'Toole, and Dr Nyan Du for their role in data collection for this study.

\section{References}

[1] Agrawal R, Sood KC, Agarwal B. Evaluation of Early versus Delayed Laparoscopic Cholecystectomy in Acute Cholecystitis. Surg Res Pract. 2015; 2015: 349801.

[2] Okamoto K, Suzuki K, Takada T, Strasberg SM, Asbun HJ, Endo I, et al. Tokyo Guidelines 2018: flowchart for the management of acute cholecystitis. J Hepatobiliary Pancreat Sci. 2018; 25 (1): 55-72.

[3] De Mestral C, Rotstein OD, Laupacis A, Hoch JS, Zagorski B, Alali AS, et al. Comparative operative outcomes of early and delayed cholecystectomy for acute cholecystitis: a populationbased propensity score analysis. Ann Surg. 2014; 259 (1): 105 .

[4] NICE. Diagnosis and management of cholelithiasis, cholecystitis and choledocholithiasis London2014 [Available from: https: //www.nice.org.uk/guidance/cg188/evidence/fullguideline-pdf-193302253.

[5] Gul R, Dar RA, Sheikh RA, Salroo NA, Matoo AR, Wani SH. Comparison of early and delayed laparoscopic cholecystectomy for acute cholecystitis: experience from a single center. N Am J Med Sci. 2013; 5 (7): 414-8.

[6] Gurusamy KS, Davidson C, Gluud C, Davidson BR. Early versus delayed laparoscopic cholecystectomy for people with acute cholecystitis. Cochrane Database Syst Rev. 2013 (6): Cd005440.

[7] Zhou MW, Gu XD, Xiang JB, Chen ZY. Comparison of clinical safety and outcomes of early versus delayed laparoscopic cholecystectomy for acute cholecystitis: a metaanalysis. ScientificWorldJournal. 2014; 2014: 274516.

[8] Wu XD, Tian X, Liu MM, Wu L, Zhao S, Zhao L. Metaanalysis comparing early versus delayed laparoscopic cholecystectomy for acute cholecystitis. Br J Surg. 2015; 102 (11): 1302-13.
[9] Ozkardeş AB, Tokaç M, Dumlu EG, Bozkurt B, Ciftçi AB, Yetişir F, et al. Early versus delayed laparoscopic cholecystectomy for acute cholecystitis: a prospective, randomized study. Int Surg. 2014; 99 (1): 56-61.

[10] Sutton AJ, Vohra RS, Hollyman M, Marriott PJ, Buja A, Alderson D, et al. Cost-effectiveness of emergency versus delayed laparoscopic cholecystectomy for acute gallbladder pathology. Br J Surg. 2017; 104 (1): 98-107.

[11] Kao LS, Ball CG, Chaudhury PK. Evidence-based Reviews in Surgery: Early Cholecystectomy for Cholecystitis. Ann Surg. 2018; 268 (6): 940-2.

[12] Khalid S, Iqbal Z, Bhatti AA. Early Versus Delayed Laparoscopic Cholecystectomy For Acute Cholecystitis. J Ayub Med Coll Abbottabad. 2017; 29 (4): 570-3.

[13] Bokhari S, Walsh U, Qurashi K, Liasis L, Watfah J, Sen M, et al. Impact of a dedicated emergency surgical unit on early laparoscopic cholecystectomy for acute cholecystitis. Ann R Coll Surg Engl. 2016; 98 (2): 107-15.

[14] Suhardja TS, Bae L, Seah EZ, Cashin P, Croagh DG. Acute surgical unit safely reduces unnecessary after-hours cholecystectomy. Ann R Coll Surg Engl. 2015; 97 (8): 568-73.

[15] Kaya C, Yang PF, Wong SW, Truskett PG. Outcomes of an acute care surgery model: a 10-year follow-up study. ANZ J Surg. 2020; 90 (3): 257-61.

[16] Yokoe M, Hata J, Takada T, Strasberg SM, Asbun HJ, Wakabayashi G, et al. Tokyo Guidelines 2018: diagnostic criteria and severity grading of acute cholecystitis (with videos). J Hepatobiliary Pancreat Sci. 2018; 25 (1): 41-54.

[17] Lehane CW, Jootun RN, Bennett M, Wong S, Truskett P. Does an acute care surgical model improve the management and outcome of acute cholecystitis? ANZ J Surg. 2010; 80 (6): 438-42.

[18] Wiggins T, Markar SR, MacKenzie H, Faiz O, Mukherjee D, Khoo DE, et al. Optimum timing of emergency cholecystectomy for acute cholecystitis in England: population-based cohort study. Surg Endosc. 2019; 33 (8): 2495-502.

[19] Altieri MS, Brunt LM, Yang J, Zhu C, Talamini MA, Pryor AD. Early cholecystectomy $(<72 \mathrm{~h})$ is associated with lower rate of complications and bile duct injury: a study of 109,862 cholecystectomies in the state of New York. Surg Endosc. 2020; 34 (7): 3051-6. 